This is a self-archived version of an original article. This version may differ from the original in pagination and typographic details.

Author(s): Dutta, Arpan

Title: Ytterbium-doped fibers for high-power fiber lasers

Year: 2021

Version: Accepted version (Final draft)

Copyright: @ 2021 Author(s). Published by AIP Publishing.

Rights: In Copyright

Rights url: http://rightsstatements.org/page/lnC/1.0/?language=en

Please cite the original version:

Dutta, A. (2021). Ytterbium-doped fibers for high-power fiber lasers. In M.M. Sinha, \& S.S. Verma (Eds.), AMRP-2020 : 5th National e-Conference on Advanced Materials and Radiation Physics (Article 020071). American Institute of Physics. AIP Conference Proceedings, 2352. https://doi.org/10.1063/5.0052378 


\title{
Ytterbium-Doped Fibers for High-Power Fiber Lasers
}

\author{
Arpan Dutta ${ }^{1, \text { a) }}$ \\ ${ }^{1}$ Nanoscience Center and Department of Physics, P. O. Box 35, 40014 University of Jyväskylä, Finland

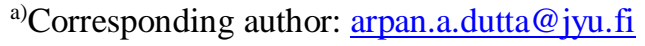

\begin{abstract}
Ytterbium (Yb) doped optical fibers are widely used in high-power applications and ultrafast lasing since they show adequate power-handling capability and provide desirable beam quality. Yb-doped fibers with large core area can support high power but often act as a multimode fiber and compromise the output beam quality. Hence, it is important to attain a proper balance between the power-handling capability and the beam quality. Yb-doped fibers as a gain medium in pulsed fiber laser systems are prone to nonlinear optical effects due to the presence of high peak power in the ultrashort pulses. Nonlinearity such as self phase modulation (SPM) affects the width and the shape of the pulse, both temporally and spectrally, by inducing chirp during its propagation along the fiber. In this work, finite element method was employed to compute linearly polarized transverse modes and the corresponding modal powers of Yb-fibers with different core areas to optimize the trade-off between the power-handling capability and the beam quality. The optimal fiber was implemented as a gain medium in a passively mode-locked fiber laser system to generate an ultrashort picosecond pulse. The spectral width of the picosecond pulse was studied as a function of pump power to spot the presence of SPM and chirp. To grow a better understanding on the chirped pulse propagation in the fiber, nonlinear Schrödinger equation was numerically simulated in the anomalous dispersion regime under the influence of initial chirp. The simulations reveal a strategy to compress a pulse temporally and utilize it in all-fiber chirped pulse amplification to mitigate nonlinearity in high power applications.
\end{abstract}

\section{INTRODUCTION}

Ytterbium $(\mathrm{Yb})$ doped fibers with large core area are preferable for high-power fiber lasers since they can provide desirable power-handling capability and beam quality in lasing [1]. Such fibers typically consist a core region (doped with $\mathrm{Yb}$ ) as gain medium and one or multiple cladding for pump guidance [2]. These fibers can support high power due to their large mode area (LMA) and further increment in their core size can improve their power handling capability. However, due to larger core, often the fibers yield multimode propagation and thereby compromise the output beam quality [1]. Hence, a balance between power-handling capability and beam quality is important.

Yb-fibers are widely utilized in mode-locked fiber lasers for ultrashort pulse generation [2]. Such high power applications, however, often challenged by the nonlinear optical effects [1]. Since the ultrashort pulses carry high peak powers through a tightly confined light path in the fiber core, the fiber started to incur limitations in its performance due to the nonlinearities induced by the high power and the generated pulses become chirped [3]. Application of chirped pulse amplification (CPA) system can mitigate the nonlinearity by stretching the pulse before amplification (i.e. minimizing its peak power) while compressing its amplified version [4]. LMA fibers can also be utilized in the latter part i.e. as a pulse compressor in an all-fiber CPA system since the chirped pulse propagation in an optical fiber engenders decrement in the temporal width of the pulse in the negative dispersion (anomalous) regime [5].

In this work, the power-handling capability of Yb-fiber is studied with the help of finite element method (FEM). An ultrashort pico-second pulse is experimentally generated using a mode-locked Yb-doped fiber laser system and the presence of chirp is shown in the generated pulse. Chirped pulse propagation and compression in the anomalous dispersion regime under the influence of initial chirp is also studied numerically using the nonlinear Schrödinger equation (NLSE) for Yb-doped optical fibers. 


\section{POWER-HANDLING CAPABILITY OF YB-DOPED FIBER}

Mode analysis and modal power calculations were done for three different $\mathrm{Yb}$-doped step-index fibers having core diameters $5.3 \mu \mathrm{m}, 12.5 \mu \mathrm{m}$ and $25.0 \mu \mathrm{m}$ with the help of a commercial FEM package (COMSOL Multiphysics v. 5.5). The fibers were modelled in 2D ( $x y$ plane) by assuming that they are uniformly elongated in $z$-direction. The refractive index of core $\left(n_{\text {core }}\right)$ and the numerical aperture (NA) were 1.46 and 0.1 , respectively, for all fibers. The operating wavelength was $1064 \mathrm{~nm}$ in the computation since standard Yb-doped fibers are operated around $1 \mu \mathrm{m}$ in lasing [2].

The FEM simulations for a single fiber provided the effective refractive indices $\left(n_{\text {eff }}\right)$ for all possible modes present in that fiber and each of those modes had an unique $n_{\text {eff }}$, which was greater than the refractive index of clad ( $\left.n_{\text {clad }}\right)$, but lower than $n_{\text {core }}$. Mode-specific normalized propagation constants $(b)$ were then calculated from the obtained $n_{\text {eff }}$ values since mathematically, $b=\left(n_{\text {eff }}^{2}-n_{\text {clad }}^{2}\right) / \mathrm{NA}^{2}[6]$.

Modal power equations for a fiber optic waveguide provide information of the power carried in the core $\left(\mathrm{P}_{\text {core }}\right)$ and in the clad $\left(\mathrm{P}_{\text {clad }}\right)$ for a specific mode [6]. The computed values of $b$ along with the known $V$ number of the fiber were then employed in the modal power equations to calculate $\mathrm{P}_{\text {core }}$ and $\mathrm{P}_{\text {clad }}$ as -

$$
\begin{gathered}
\mathrm{P}_{\text {core }}=C \pi a^{2}\left[1-\left\{J_{l-1}(U) J_{l+1}(U) / J_{l}^{2}(U)\right\}\right], \\
\mathrm{P}_{\text {clad }}=C \pi a^{2}\left[\left\{K_{l-1}(W) K_{l+1}(W) / K_{l}^{2}(W)\right\}-1\right],
\end{gathered}
$$

where $a$ is the core radius, $U=V \sqrt{1-b}, W=V \sqrt{b}, J_{l}$ and $K_{l}$ are the Bessel $\mathrm{J}$ and Bessel $\mathrm{K}$ functions correspond to the linearly polarized $\left(\mathrm{LP}_{\mathrm{lm}}\right)$ transverse modes of the fiber, and $C$ is an arbitrary constant [6]. As the core diameter of the fibers increased from $5.3 \mu \mathrm{m}$ to $25.0 \mu \mathrm{m}$, the fibers became multimode. Hence, the ratio between the total core and the total clad power i.e. $\sum \mathrm{P}_{\text {core }} / \sum \mathrm{P}_{\text {clad }}$ was studied with respect to the core diameter where the $\sum$ sign includes all possible modes supported by the fiber. Figure 1(a) represents $\sum \mathrm{P}_{\text {core }} / \sum \mathrm{P}_{\text {clad }}$ as a function of core diameter in the case of Yb-doped optical fibers.

The quality of the output beam from a fiber depends on the number of modes propagating in the fiber. The higher the number of modes, the lower the beam quality [1]. The Yb-doped fiber with $5.3 \mu \mathrm{m}$ core diameter $(V=2.1908)$ supported only one fundamental mode $\left(\mathrm{LP}_{01}\right)$ at $1064 \mathrm{~nm}$ and since it is single mode, the $\sum \mathrm{P}_{\text {core }}$ was the contribution of the $\mathrm{LP}_{01}$ mode only. The $\mathrm{Yb}$-doped fiber with $12.5 \mu \mathrm{m}$ core diameter $(V=3.6908)$ can carry one fundamental $\left(\mathrm{LP}_{01}\right)$ and only one higher order mode $\left(\mathrm{LP}_{11}\right)$ at $1064 \mathrm{~nm}$. Therefore, it can be called as 'few-mode' fiber where both $\mathrm{LP}_{01}$ and $\mathrm{LP}_{11}$ contributed in the $\sum \mathrm{P}_{\text {core }}$. In the case of $\mathrm{Yb}$-doped fiber with $25 \mu \mathrm{m}$ core diameter $(V=7.3816)$, seven higher order modes were found $\left(\mathrm{LP}_{11}, \mathrm{LP}_{21}, \mathrm{LP}_{02}, \mathrm{LP}_{31}, \mathrm{LP}_{12}, \mathrm{LP}_{41}\right.$ and $\left.\mathrm{LP}_{22}\right)$ along with the fundamental mode $\left(\mathrm{LP}_{01}\right)$ at $1064 \mathrm{~nm}$ and all of them contributed in the $\sum \mathrm{P}_{\text {core }}$ as expected from a multimode fiber. Since the fundamental mode of the fiber provides the best possible beam quality among all modes, the ratio between the power carried by the fundamental mode $\left(\mathrm{P}_{\text {fundamental }}\right)$ and the power carried by all other higher order modes $\left(\mathrm{P}_{\text {higher }}\right)$, i.e. $\mathrm{P}_{\text {fundamental }} / \sum \mathrm{P}_{\text {higher }}$, can be used to estimate (qualitatively) the output beam quality. Figure 1(b) depicts the aforementioned quantification of beam quality in terms of core diameter for the two multimode Yb-doped active fibers (core diameters $12.5 \mu \mathrm{m}$ and $25.0 \mu \mathrm{m}$ ) while the fiber with core diameter $5.3 \mu \mathrm{m}$ was excluded since it worked as a single mode fiber at $1064 \mathrm{~nm}$. The pie charts in Fig. 1(b) next to the data points represent the percentage contribution of each mode in $\sum \mathrm{P}_{\text {core }}$ of the corresponding fiber. 


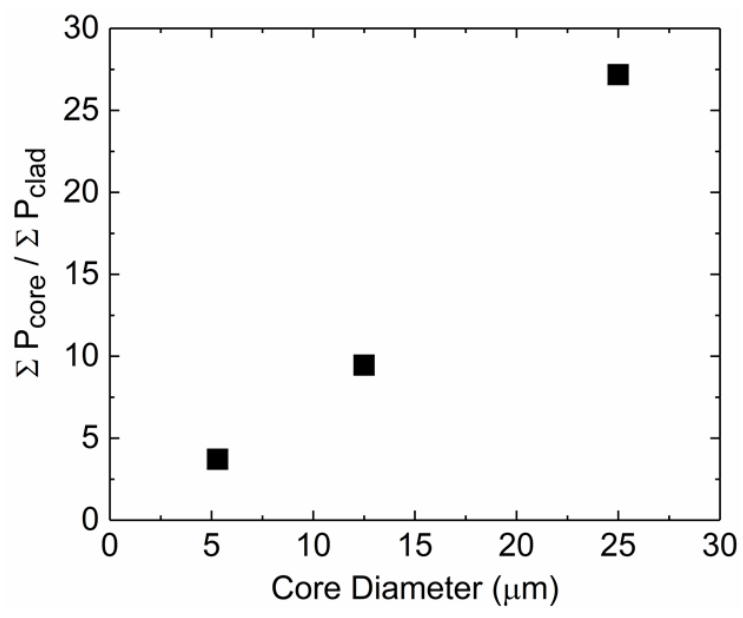

(a)

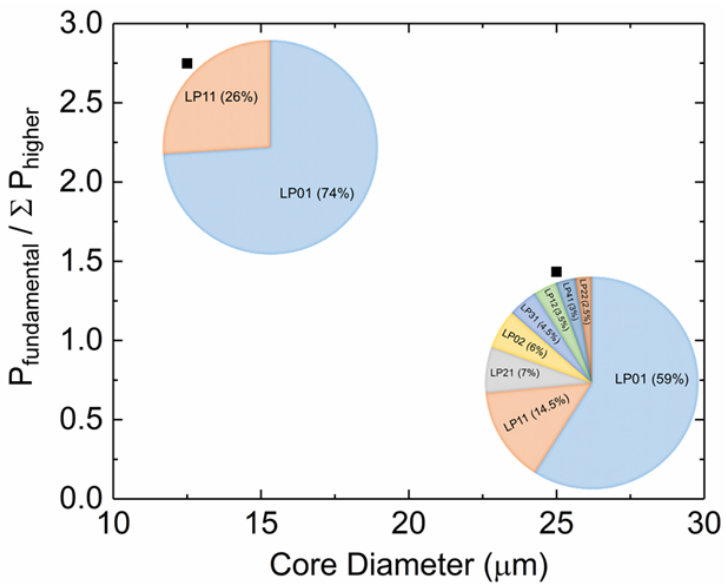

(b)

FIGURE 1. (a) Ratio between the total core and the total clad power as a function of core diameter of the fiber (b) Ratio between the power carried by the fundamental mode and the power carried by all other higher order modes as a function of core diameter of the fiber. The pie charts next to the data points represent the percentage contribution of each mode in $\sum \mathrm{P}_{\text {core }}$ of the corresponding fiber

The fiber that can provide high power-handling capability and acceptable beam quality is suitable for high-power fiber laser applications. From Fig. 1 it is clear that the fiber with core diameter $5.3 \mu \mathrm{m}$ is best in terms of beam quality since it is a single mode fiber but it has the lowest power-handling capability among the three fibers. On the other hand, the fiber with core diameter $25 \mu \mathrm{m}$ may have the highest power-handling capability but it has lowest power in its fundamental mode among the three fibers and hence, yields the poorest beam quality. Therefore, the Yb-doped fiber having a core diameter of $12.5 \mu \mathrm{m}$ will be the optimal choice for lasing applications by maintaining a reasonable trade-off between the power-handling capability and the beam quality.

\section{CHIRPED PULSE GENERATION}

The optimum Yb-doped fiber (core diameter: $12.5 \mu \mathrm{m}$ ) having a length of $0.8 \mathrm{~m}$ was then implemented in a (passive) mode-locked fiber laser system as the active medium where a semiconductor laser diode (JDSU, $250 \mathrm{~mW}$ ) was used for pumping at $976 \mathrm{~nm}$. A semiconductor saturable absorber mirror (Batop) and a fiber Bragg grating (TeraXion, reflectivity $50 \%$ at $900-1100 \mathrm{~nm}$ ) made the resonator cavity. The output pulse was collected through the fiber Bragg grating and its spectral width $(\Delta \omega)$ was measured $\left(\lambda_{\mathrm{FWHM}}\right.$ in wavelength scale) by an optical spectrum analyzer. The temporal width $(\Delta t)$ of the pulse was traced with the help of an autocorrelator.

In the experiment, temporal and spectral widths of the output pulse were recorded for different pump powers (80 $\mathrm{mW}-200 \mathrm{~mW}$ ). Insignificant variation was found in the temporal width of the output pulse with the varying pump power. The temporal pulse width was $2361.0 \mathrm{fs}$ or $2.361 \mathrm{ps}$ at $150 \mathrm{~mW}$ pump power and varied $\pm 496.7 \mathrm{fs}$ for pump powers $80 \mathrm{~mW}$ to $200 \mathrm{~mW}$. The change in the spectral width of the output pulse, however, was dramatic and it implies the presence of nonlinear effects in the fiber. Figure 2(a) reports the $\lambda_{\text {FWHM }}$ of the output pulse for various pump powers from $80 \mathrm{~mW}$ to $200 \mathrm{~mW}$.

From Fig. 2(a) it can be concluded that the increment in pump power increases the peak power of the output pulse, which in turn induces self phase modulation (SPM), a nonlinear optical effect in which new frequency components are generated under the pulse envelope as it propagates through the fiber [7]. The new frequency components generated due to the SPM effect broaden the pulse in the spectral domain while the temporal pulse width remains almost identical during the pulse propagation. Such finding experimentally shows the crucial role of nonlinearity in high power fiber optic applications.

SPM also influences the pulse shape during the pulse propagation through the fiber by inducing 'chirp'. Chirp is an instantaneous frequency change in the pulse envelope. SPM-induced chirp increases in magnitude along the propagation path and deviates the shape of the spectrum [7]. Deviation of pulse shape can be identified through the time-bandwidth product (TBP) of the pulse, which is a product of the temporal and the spectral pulse widths, i.e. 
$\mathrm{TBP}=\Delta \omega \times \Delta t$. A transform-limited pulse does not deviate from its initial pulse shape during the propagation and maintains its TBP along the propagation path. For a specific pulse shape, the TBP is constant. The TBP values are 0.4413 and 0.3148 for a Gaussian and hyperbolic-secant pulse shape, respectively [8]. The output pulse of the modelocked fiber laser had pulse widths $\Delta t=2.361 \mathrm{ps}$ and $\Delta \omega=2.7824 \times 10^{11} \mathrm{~Hz}$ at $150 \mathrm{~mW}$ pump power and the corresponding TBP value was 0.6569 . The higher value of TBP in the laser output than that of the transform-limited pulses confirmed that the pulse was not transform limited and hence, chirped. A chirped pulse deviates from its initial pulse shape during the propagation through the fiber [7].

\section{CHIRPED PULSE COMPRESSION}

Chirped pulse propagation in the high-power active fibers mostly influenced by the group velocity dispersion and various nonlinear optical effects. Dispersion affects the pulse temporally while nonlinearity (like SPM) affects it spectrally [7]. Chirped pulse can also be utilized in all-fiber CPA system since the temporal width of a chirped pulse can be compressed in the anomalous dispersion regime [5].

To have an insight on the chirped pulse propagation (and compression) in a fiber optic system, NLSE was numerically solved using the split step Fourier method (implemented by MATLAB) where the mathematical form of NLSE was considered as -

$$
i \frac{\partial A(z, T)}{\partial z}=-\frac{i \alpha A(z, T)}{2}+\frac{\beta_{2}}{2} \frac{\partial^{2} A(z, T)}{\partial T^{2}}-\gamma|A(z, T)|^{2} A(z, T),
$$

and the three terms in the right hand side represent successively - fiber loss $(\alpha)$, dispersion $\left(\beta_{2}\right)$ and nonlinearity $(\gamma)$. The temporal and spectral shapes of a Gaussian pulse (at $z=0)$ were considered as -

$$
\begin{gathered}
A(0, T)=\exp \left[-(1+i C) T^{2} / 2 T_{0}^{2}\right] \\
A(0, \omega)=\left[2 \pi T_{0}^{2} /(1+i C)\right]^{1 / 2} \exp \left[-\omega^{2} T_{0}^{2} / 2(1+i C)\right],
\end{gathered}
$$

where $T_{0}$ is the initial temporal width of the pulse, $C$ is the chirp parameter and was varied in the numerical simulations. Nonlinearity (SPM) only affects the pulse spectrally by inducing chirp in it and the chirp-dependent spectral width of the pulse is $\Delta \omega=\sqrt{1+C^{2}} / T_{0}$. Since SPM alone does not affect the pulse temporally (i.e. $T_{0}$ is independent of chirp), in the absence of chirp (i.e. $C=0$ ), the pulse is transform-limited $\left(\Delta \omega T_{0}=1\right)$ [7].

Dispersion plays a critical role in the temporal evolution of a chirped pulse. If both the dispersion and the chirp are positive (i.e. normal dispersion regime with $\beta_{2} C>0$ ), the chirped Gaussian pulse broadens temporally during its propagation because dispersion-induced chirp become additive with the initial chirp. A positively chirped pulse under the anomalous dispersion regime (i.e. $\beta_{2}$ is negative, $C$ is positive, $\beta_{2} C<0$ ), however, experiences compression in its temporal width along the propagation distance and attains a minimum value of the temporal width $T_{\min }=T_{0} / \sqrt{1+C^{2}}$ at a certain distance. In this case, the initial spectral width of the pulse $\left(\omega_{0}\right)$ remains almost identical during the propagation and when the pulse attains its minimum temporal width $\left(T_{\min }\right)$, the chirp becomes zero $(C=0)$ as well as the pulse becomes transform-limited $\left(\omega_{0} T_{\min }=1\right)$. This happens because the dispersion-induced chirp is negative in the anomalous regime (since $\beta_{2}$ is negative) and it neutralizes the initial positive chirp [7].

Chirped pulse propagation and compression in the anomalous dispersion regime under the influence of initial positive chirp were studied numerically by varying the chirp parameter $C$. The spectral and temporal widths of the pulse were calculated for multiple chirp parameters over a normalized propagation distance $z / L_{D}$ where the dispersion length $L_{D}$ is defined as $L_{D}=T_{0}^{2} /\left|\beta_{2}\right|$ [7]. Figure 2(b) reports the evolution of temporal width of the pulse over the normalized propagation distance for different positive chirps $(C=0.5,1$ and 2$)$. The spectral width of the pulse was found unaffected along the propagation distance for all chirp parameters. 


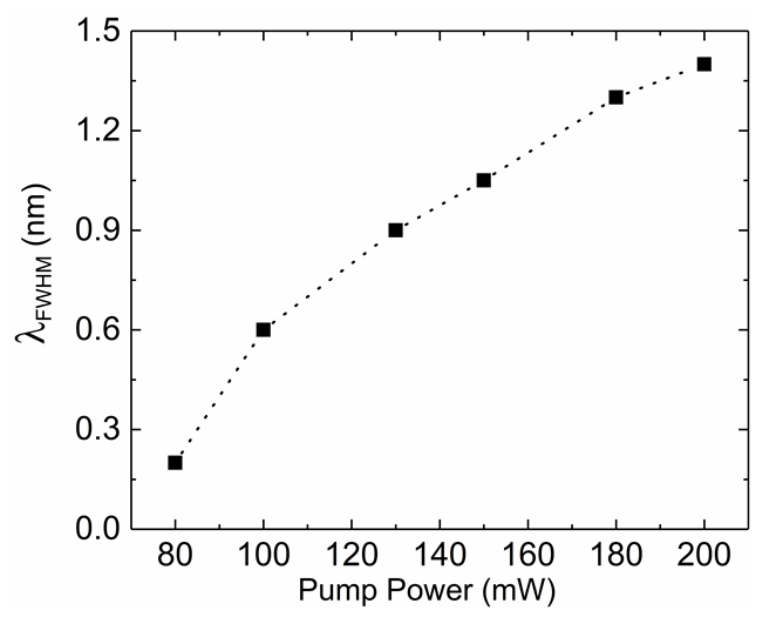

(a)

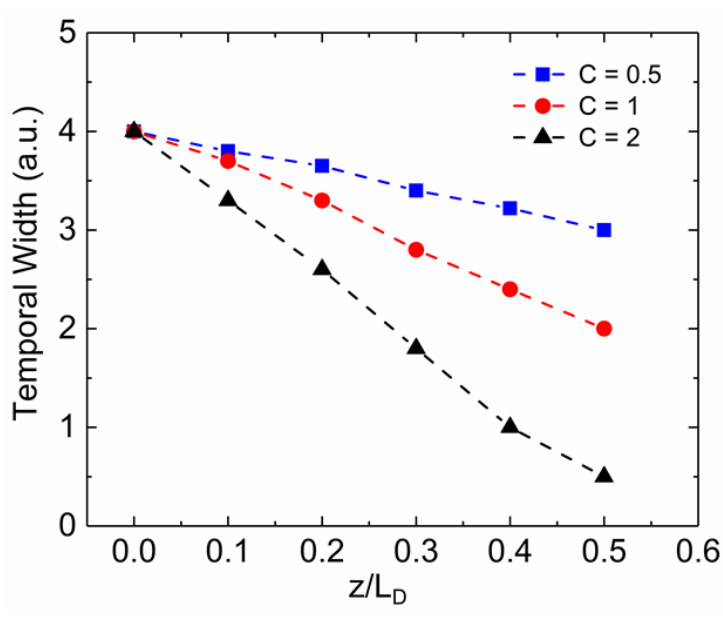

(b)

FIGURE 2. (a) Spectral width of the output pulse (2.361 ps) of the mode-locked fiber laser for different pump powers (b) Evolution of the temporal width of a chirped Gaussian pulse for different positive chirps

According to Fig. 2(b), a positively chirped pulse experiences temporal compression without any spectral change while propagating under the anomalous dispersion. The more the pulse is positively chirped, the more it compressed temporally. Since the ultrashort high-power pulses generated from a mode-locked fiber laser are often chirped and stretched in a CPA system before amplification, suitably designed Yb-fibers operating in anomalous dispersion can be used to compress the amplified pulses to their ultrashort width.

\section{CONCLUSIONS}

Mode analysis and modal power calculations were done using FEM for Yb-doped fibers having different core diameters. The fiber with core diameter $12.5 \mu \mathrm{m}$ was found optimal for lasing in terms of power-handling capability and beam quality. The optimum fiber was then employed as an active medium in a passively mode-locked fiber laser system to generate an ultrashort $2.361 \mathrm{ps}$ pulse. The spectral width of the picosecond pulse was studied as a function of pump power and the presence of SPM and chirp in the laser output was identified. To build an insight on the nature of the chirped pulse and its applicability in an all-fiber CPA system, numerical simulations based on NLSE were carried out in the anomalous dispersion regime under the influence of initial chirp. The numerical outcomes revealed that a positively chirped pulse can be compressed in the temporal domain, along its propagation length inside the fiber, in the presence of anomalous dispersion. Such findings provide essential information about Yb-doped optical fibers and their performance in high-power fiber laser systems.

\section{ACKNOWLEDGMENTS}

The author gratefully acknowledge Dr. Mrinmay Pal (Central Glass and Ceramic Research Institute, Kolkata, India) for his valuable guidance and cooperation during the research work.

\section{REFERENCES}

1. F. Stutzki, F. Jansen, H. Otto, C. Jauregui, J. Limpert and A. Tünnermann, Optica 1(4), 233-242 (2014).

2. D. J. Richardson, J. Nilsson and W. A. Clarkson, J. Opt. Soc. Am. B 27(11), B63-B92 (2010).

3. C. Jauregui, J. Limpert and A. Tünnermann, Nature Photon 7, 861-867 (2013).

4. M. E. Fermann and I. Hartl, Nature Photon 7, 868-874 (2013).

5. S. V. Smirnov, S. M. Kobtsev and S. V. Kukarin, Opt. Express 23(4), 3914-3919 (2015).

6. A. Ghatak and K. Thyagarajan, An Introduction to Fiber Optics (Cambridge University Press, Cambridge, 1998).

7. G. P. Agrawal, Nonlinear Fiber Optics (Academic Press, Cambridge, 2012).

8. A. Webster, Useful Mathematical Formulas for Transform Limited Pulses (Accessed 30 September 2020). 